\title{
Synthesis of polymer-vesicle latex particles through an ATRP-based approach
}

\author{
Olessya P. Loiko' · Johan P. A. Heuts ${ }^{2}$ - Alex M. van Herk ${ }^{3}$
}

Received: 26 November 2021 / Revised: 11 January 2022 / Accepted: 11 January 2022 / Published online: 10 February 2022

(c) The Author(s) 2022

\begin{abstract}
The synthesis of polymer-vesicle latex particles by using extruded vesicles of dimethyldioctadecyl ammonium bromide (DODAB) via an atom transfer radical polymerization-based approach is reported. Cryo-TEM characterization revealed the growth of polymer from one side of the vesicle, and the final morphology of the resulting particles was affected by monomer feed composition. Protrusions or nanocapsules were synthesized when MMA:BA:EGDMA or MMA were used, respectively. Furthermore, it was found that the co-oligomer composition and chain length affect the resulting morphologies of the particles.
\end{abstract}

Keywords Polymer vesicles · Polymerization · Atom transfer radical polymerization (ATRP)

\section{Introduction}

Vesicles have attracted an increasing interest due to their potential application as carriers for active ingredients and controlled release systems [1-5]. In water, vesicle structures result from self-assembly of amphiphilic molecules above the gel-to-liquid crystalline phase transition temperature [6]. As a result, a closed spherical bilayer which has a water containing compartment inside is produced. Nowadays, the class of double-tailed quaternary ammonium salts is being widely used to form vesicles due to their commercial availability [7-10].

Vesicle-templating polymerization can be conducted via polymerization on the outside of the vesicle $[2,7]$ or by a

Johan P. A. Heuts

j.p.a.heuts@tue.nl

$\triangle$ Alex M. van Herk

a.m.v.herk@tue.nl

1 Avery Dennison Label and Graphic Materials, Oegstgeest, The Netherlands

2 Department of Chemical Engineering \& Chemistry and Institute for Complex Molecular Systems, Eindhoven University of Technology, PO Box 513, 5600 MB Eindhoven, The Netherlands

3 Department of the Built Environment, Eindhoven University of Technology, PO Box 513, 5600 MB Eindhoven, The Netherlands layer-by-layer approach $[1,11]$. The latter has been extensively used to produce polymeric nanocapsules for biomedical applications $[12,13]$. The drawback of this technique is that in order to obtain the necessary shell thickness, several layers should be deposited which results in a tedious procedure. Moreover, the polyelectrolytes are absorbed via electrostatic interactions, and, therefore, the addition of a crosslinker is often required to make more robust structures [14].

Successful synthesis of polymeric nanocapsules was reported by Ali et al. using RAFT-mediated starved-feed emulsion polymerization $[7,8]$. This method was based on the previously applied strategy to encapsulate Gibbsite platelets by the same author [15] and other inorganic particles by Hawkett et al. [16-19] Briefly, first a small polyelectrolyte consisting of butyl acrylate (BA) and acrylic acid (AA) units (i.e., $\mathrm{BA}_{6}-\mathrm{co}_{-} \mathrm{AA}_{9}$ ) is synthesized through reversible addition-fragmentation chain transfer (RAFT) polymerization and adsorbed on the surface of the vesicles. RAFT moieties of the polyelectrolyte then allow for further chain extension upon injection of initiator (V-501), and addition of monomer mixtures (e.g., methyl methacrylate (MMA)/ BA) under starved feed conditions. Ali et al. also extended this approach towards $\mathrm{pH}$-responsive nanocapsules $[8,20]$, and studies into their potential application as a drug delivery system were conducted [20].

In this work, we explore the application of the earlier developed ATRP-based approach on vesicle polymerization. For this purpose, we have chosen dimethyldioctadecyl 

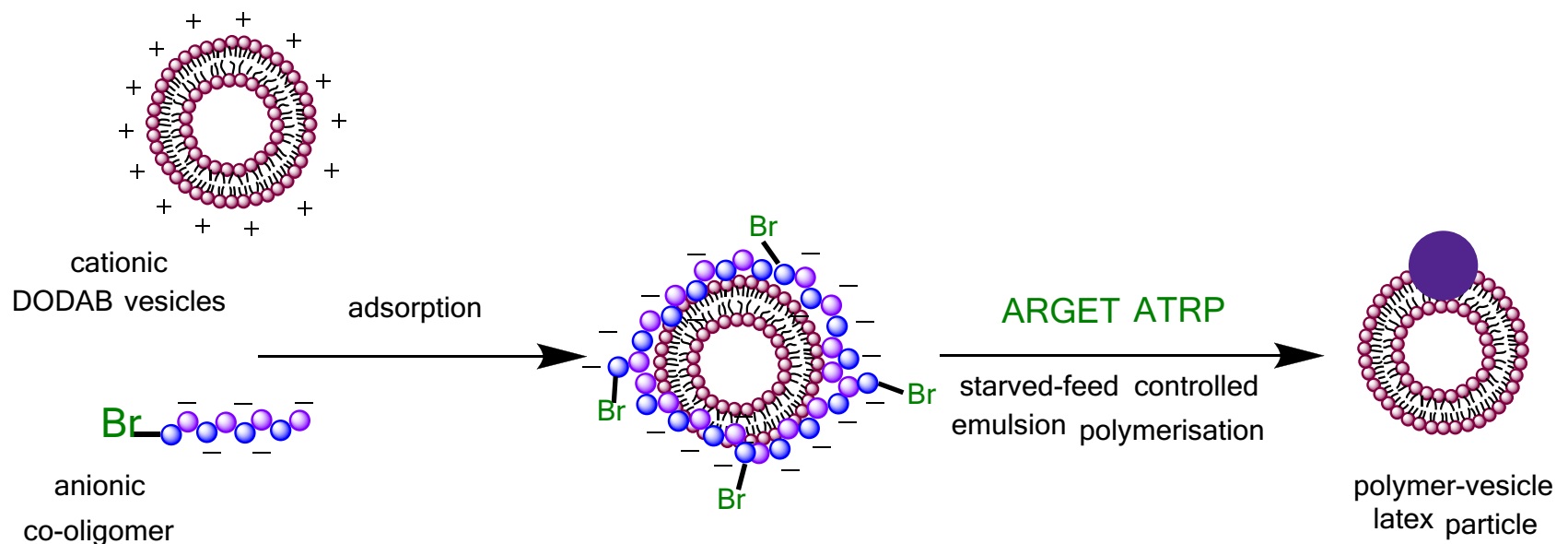

Scheme 1 Schematic representation of the synthesis of polymervesicle latex particles by aqueous starved feed emulsion polymerization using ATRP co-oligomers. The same co-oligomers and polymerization conditions were previously used for the synthesis of polymer-Gibbsite nanocomposites [21]. The thickness of DODAB vesicle bilayer is around $5 \mathrm{~nm}^{8}$ ammonium bromide (DODAB) vesicles, since they were previously used in a similar RAFT-based method [7, 8].

Anionic co-oligomers $\mathrm{BA}_{n}-$ co-AA $\mathrm{A}_{m}$ were synthesized by atom transfer radical polymerization (ATRP) and used as stabilizers for the initial template and served as macroinitiators in subsequent ARGET ATRP starved-feed emulsion polymerization (Scheme 1).

Through this work, we will be able to see whether the RAFT moieties on the anionic co-oligomers as compared to the co-oligomers prepared via ATRP make a difference in the obtained morphologies and whether other controlled polymerization techniques will lead to similar results. Also, in some cases, a sulphur-free capsule is desired. As will be shown, cryo-TEM characterization of the obtained composite latex particles showed similar morphologies as reported before in RAFT-based systems [15]. As in these RAFT-based systems, the final morphologies were found to depend on monomer feed composition, and on co-oligomer composition and chain length, we also investigated these effects in the current ATRP systems.

\section{Experimental}

\section{Materials}

Butyl acrylate (BA, Aldrich, 99\%), ethylene glycol dimethacrylate (EGDMA, Aldrich, 98\%), and methyl methacrylate (MMA, Aldrich, 99\%) were purified by passing them through a column filled with inhibitor removal. $\mathrm{CuBr}_{2}$ (99.999\%, Aldrich), ascorbic acid (99\%, Aldrich), tris(2-pyridylmethyl) amine (TPMA, 99\%, Aldrich), N,N-dimethylformamide (DMF), and $\mathrm{NaCl}$ (99\%, Merck) were used as received.

\section{Characterization}

Molar mass distributions, the number average molar mass $\left(M_{\mathrm{n}}\right)$, and dispersity $(\nexists)$ of the anionic co-oligomer and the composite latexes were measured by size exclusion chromatography (SEC) using a Waters SEC set-up equipped with a Waters model 510 pump, a model 410 differential refractometer, and a 2487 dual $\lambda$ absorbance detector (operated at $\lambda=254 \mathrm{~nm}$ ). A set of two mixed bed columns (Mixed-C, Polymer Laboratories, $30 \mathrm{~cm}, 40{ }^{\circ} \mathrm{C}$ ) was used. Tetrahydrofuran was used as the eluent, and the system was calibrated using polystyrene standards $\left(\right.$ range $\left.=580-7.5 \times 10^{6} \mathrm{~g} \mathrm{~mol}^{-1}\right)$. Data analysis was performed using the software Empower Pro version 2 from the Waters Corporation.

${ }^{1} \mathrm{H}$ NMR spectra were recorded on a Varian $400 \mathrm{MHz}$ spectrometer using chloroform-d and water- $\mathrm{d}_{2}$ as solvents. Analysis of the spectra was done using the software MestReNova 9.0.0-12,821 from Mestrelab Research S.L.

The particle size distribution and zeta potential $(\zeta)$ were determined at $23{ }^{\circ} \mathrm{C}$ using a Malvern Zetasizer Nano ZS instrument. The $\zeta$ potential was calculated from the electrophoretic mobility $(\mu)$ using the Smoluchowski relationship, $\zeta=\eta \mu / \varepsilon$, with $k a \gg 1$ (where $\eta$ is the viscosity, $\varepsilon$ is the dielectric constant of the medium, $k$ and $a$ are the DebyeHückel parameter and particle radius, respectively).

Cryogenic transmission electron microscopy (cryoTEM) measurements were conducted performed on FEI Titan Krios equipped with automated sample loader and Field Emission Gun (FEG) operating at $300 \mathrm{kV}$. Images were recorded with Falcon II camera (4X4) with magnification of 29,000 and pixel size of 2.873. Vitrification of the vesicles samples was carried out using a vitrification 


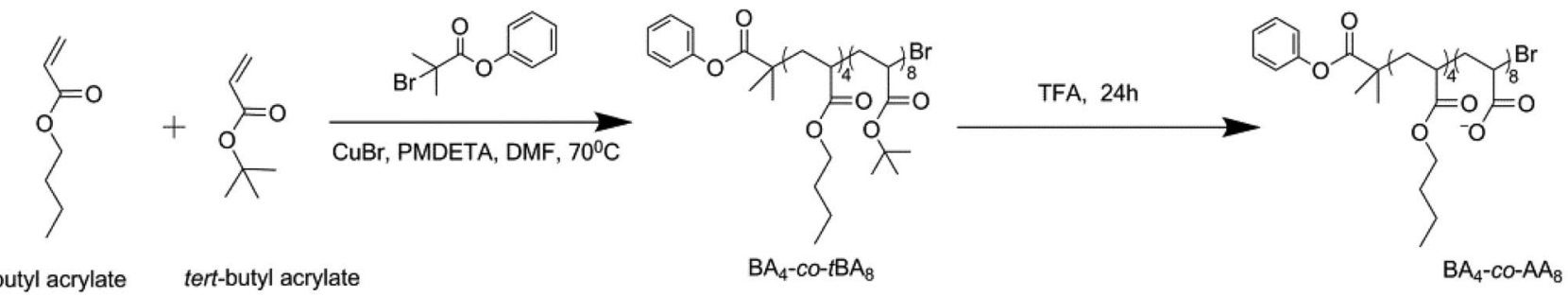

Scheme 2 Schematic representation of the synthetic strategy to anionic co-oligomer from butyl acrylate and tert-butyl acrylate

robot (FEI Vitrobot Mark IV). All samples were prepared at room temperature and $100 \%$ humidity with blotting time of $2 \mathrm{~s}$ and blot force of 1 . The sample $(5 \mu \mathrm{L})$ was applied onto a grid (Quantifoil, R2/2, Holey carbon film; freshly glow-discharged prior to use at $20 \mathrm{~mA}$ for $30 \mathrm{~s}$ ) without dilution. Excess of sample was blotted away with filter paper to leave a thin film on the grid before being vitrified in liquid ethane.

\section{Synthesis of DODAB vesicles}

DODAB vesicles were prepared by a membrane extrusion method [7]. Briefly, $10 \mathrm{mM}$ (0.63 wt\%) DODAB vesicles aqueous dispersion was mixed with $0.5 \mathrm{mM} \mathrm{NaCl}$ salt solution and heated at $65{ }^{\circ} \mathrm{C}$ in oil bath under constant stirring for at least $18 \mathrm{~h}$ prior to extrusion. The dispersion was then passed through three-stacked polycarbonate filters (Millipore, PC filters, $400 \mathrm{~nm}$ ) at $60{ }^{\circ} \mathrm{C}$ and argon pressure of 7 bar. The extrusion was repeated 5 times till narrow size distribution was achieved.

\section{General procedure for synthesis of anionic random ATRP co-oligomers}

All needles, Schlenk flask and magnetic stirring bars were dried overnight in an oven at $120{ }^{\circ} \mathrm{C}$ and purged with argon during cooling before use. The polymerization was conducted in a Schlenk flask equipped with a magnetic stirring bar. $n$-Butyl acrylate $(12.8 \mathrm{~g}, 0.1 \mathrm{~mol})$ and tert-butyl acrylate $(25.6 \mathrm{~g}, 0.2 \mathrm{~mol})$ were polymerized in $10 \mathrm{vol} \% \mathrm{DMF}$ using PMDETA $(0.26 \mathrm{~g}, 0.0015 \mathrm{~mol})$ and $\mathrm{CuBr}(0.21 \mathrm{~g}$, $0.0015 \mathrm{~mol}$ ) as a catalyst system and 2-bromo-2-methylpropionic acid phenyl ester $(0.31 \mathrm{~g}, 0.0015 \mathrm{~mol})$ as initiator using a standard procedure (Scheme 2). The reaction was carried out at $70{ }^{\circ} \mathrm{C}$ and terminated after $3 \mathrm{~h}$ by cooling the flask to $0{ }^{\circ} \mathrm{C}$ and opening it to the air. The catalyst was removed by passing the reaction mixture through a column of basic alumina. The co-oligomer was then isolated by pouring the solution into a water-methanol mixture and drying the precipitate under vacuum at room temperature. During the polymerization, samples were taken at regular intervals to measure monomer conversion using gas chromatography and molar mass distributions using SEC. Final conversion: $10 \% .{ }^{1} \mathrm{H}$ NMR $\left(400 \mathrm{MHz}, \mathrm{CDCl}_{3}\right)$ : 7.41, $7.25,7.13$ (aromatic $\mathrm{H}), 4.02\left(\mathrm{O}-\mathrm{CH}_{2}\right), 2.22\left(-\mathrm{CH}_{2}-\mathrm{CH}_{2}-\right)$, $1.82-1.52\left(-\mathrm{CH}_{2}-\mathrm{CH}_{2}-\mathrm{C}(\mathrm{C}=\mathrm{O}), 1.45\left(\mathrm{O}-\mathrm{C}\left(\mathrm{CH}_{3}\right)_{3}\right), 0.96\right.$ $\left(-\mathrm{CH}_{3}\right)$. The co-oligomer composition was determined using ${ }^{1} \mathrm{H}$ NMR from the integrated intensity ratios of the area for the aromatic protons $(\delta=7.13-7.41)$ of the end group to the area for methyl group $(\delta=0.96)$ of butyl acrylate or to the area for tert-butyl group $(\delta=1.45)$ of tert-butyl acrylate, respectively.

The co-oligomers were dissolved in dichloromethane, and a five-fold molar excess of TFA with respect to the tert-butyl group was added. The mixture was stirred at room temperature for $24 \mathrm{~h}$. The hydrolysed product was collected after rotary evaporation of the solvent and TFA. The product was then washed with dichloromethane and dried at $50{ }^{\circ} \mathrm{C}$ under vacuum [22]. The degree of hydrolysis was calculated from comparison of integrals between 0.8 and 2.8 ppm before and after treatment with TFA. No loss in $\mathrm{Br}$ end group functionality is

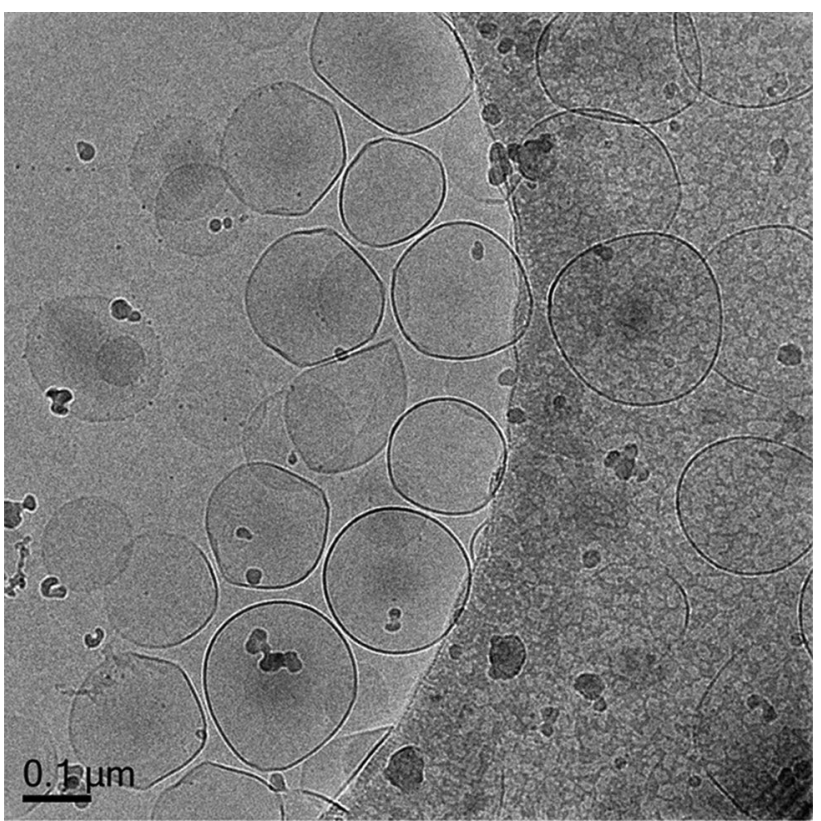

Fig. 1 Cryo-TEM image of extruded DODAB vesicles 
Table 1 Characterization of the anionic $\mathrm{BA}_{n}-c o-\mathrm{AA}_{m}$ co-oligomers

\begin{tabular}{lllrrrl}
\hline & $\boldsymbol{M}_{\mathbf{n}}{ }^{\boldsymbol{a}}\left(\mathbf{g} \cdot \mathbf{m o l}^{-\mathbf{1}}\right)$ & $\boldsymbol{\Xi}^{\boldsymbol{a}}$ & $\boldsymbol{n}^{\boldsymbol{b}}$ & $\boldsymbol{m}^{\boldsymbol{b}}$ & $\boldsymbol{X}_{\mathbf{n}}^{\boldsymbol{c}}$ & $\begin{array}{c}\mathbf{i e p}^{\boldsymbol{d}}(\mathbf{r e a c h e d} \\
\text { at a certain } \boldsymbol{\xi})\end{array}$ \\
\hline $\mathrm{BA}_{4}-\mathrm{co}-\mathrm{AA}_{8}$ & $1.4 \times 10^{3}$ & 1.15 & 4.0 & 8.3 & 12.3 & 0.35 \\
$\mathrm{BA}_{16}-\mathrm{co}-\mathrm{AA}_{32}$ & $4.5 \times 10^{3}$ & 1.28 & 16.2 & 32.3 & 48.5 & 1.35 \\
$\mathrm{BA}_{5}-\mathrm{co}-\mathrm{AA}_{5}$ & $1.2 \times 10^{3}$ & 1.17 & 5.1 & 5.3 & 10.4 & 0.25 \\
$\mathrm{BA}_{20}-\mathrm{co}-\mathrm{AA}_{20}$ & $4.2 \times 10^{3}$ & 1.30 & 20.0 & 20.2 & 40.2 & 1.20 \\
\hline
\end{tabular}

$X_{\mathrm{n}}=n+m$

${ }^{a}$ Determined by SEC against PS standards

${ }^{\mathrm{b}}$ Determined by ${ }^{1} \mathrm{H}$ NMR

${ }^{\mathrm{c}}$ Number-average degree of polymerization

${ }^{\mathrm{d}}$ Determined by zeta potential measurements expected to occur due to this treatment as no changes in the NMR spectra of the initiator ethyl $\alpha$-bromoisobutyrate were observed after the same treatment [21].

\section{Adsorption studies}

The adsorption procedure was performed as described by Ali et al. [7] In different vials, calculated amounts of ATRP co-oligomers were transferred from a 10-mM aqueous stock solution and a total volume of $10 \mathrm{~mL}$ was made by adding DDI water. Equal volumes of DODAB vesicles $(10 \mathrm{mM}$, $0.63 \mathrm{wt} \%$ ) were then added drop wise into these vials under constant stirring. The vials were kept stirring overnight at room temperature. Particle size distributions and $\zeta$ potentials were measured for these samples.

\section{Encapsulation experiments}

Vesicle templated polymerization was performed using ARGET ATRP starved feed emulsion polymerization.
Briefly, $3 \mathrm{~mL}$ of a $10-\mathrm{mM}$ aqueous stock solution of ATRP co-oligomer and $7 \mathrm{~mL}$ of DDI water were transferred into a 50-mL three neck flask after which $10 \mathrm{~mL}$ of a vesicle dispersion ( $10 \mathrm{mM}, 0.63 \mathrm{wt} \%)$ was added drop wise at a rate of $0.5 \mathrm{~mL} \min ^{-1}$ using a syringe pump 11 Elite from Harvard Apparatus under constant stirring at room temperature. A total of $1.6 \mathrm{~mL}$ of copper catalyst solution $\left(\left[\mathrm{Cu}^{2+}\right]_{0}=1.38 \times 10^{-5} \mathrm{M},\left[\mathrm{CuBr}_{2}\right]:[\mathrm{TPMA}]=1: 8\right)$ was added, and the reaction mixture was purged with argon for 30 min after which the flask was heated to $70{ }^{\circ} \mathrm{C}$. At this point, ascorbic acid was added to start the reaction, and under optimized conditions, this was done by continuous feeding of $0.3 \mathrm{~mL}$ of a $7.4 \times 10^{-5} \mathrm{M}$ aqueous ascorbic acid solution at a rate of $0.002 \mathrm{~mL} \mathrm{~min}^{-1}$ using syringe pump NE-1000 (this results in a final 1:1 molar ratio of $\left[\mathrm{CuBr}_{2}\right]_{0}$ to [ascorbic acid]). At the same time, $0.3 \mathrm{~mL}$ of deoxygenated monomer MMA at a rate $0.002 \mathrm{~mL} \mathrm{~min}^{-1}$ was fed using syringe pump 11 Elite from Harvard Apparatus. After the completion of monomer and ascorbic acid addition, the flask was kept stirring
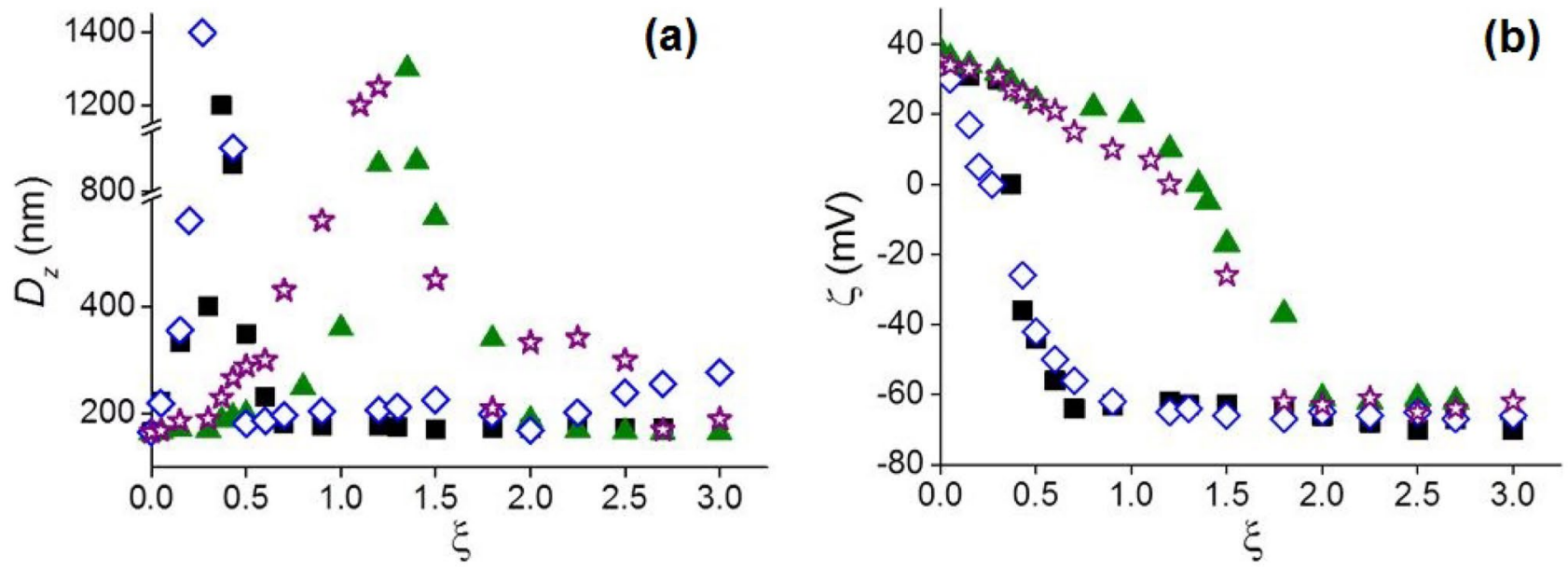

Fig. $2 D_{\mathrm{z}}$ (a) and $\zeta(\mathbf{b})$ of DODAB vesicles as a function of stoichiometric charge ratio $(\xi)$ for ATRP macroinitiator at $\mathrm{pH}=7$. Used co-oligomers: (square) $\mathrm{BA}_{4}-c o-\mathrm{AA}_{8}$, (triangle) $\mathrm{BA}_{16}-c o-\mathrm{AA}_{32}$, (diamond) $\mathrm{BA}_{5}-c o-\mathrm{AA}_{5}$, and (star) $\mathrm{BA}_{20}-c o-\mathrm{AA}_{20}$ 


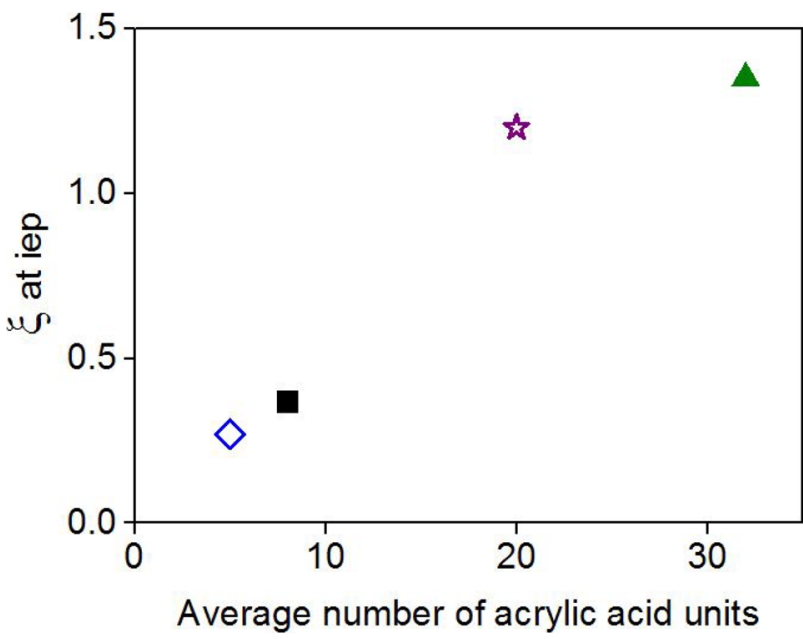

Fig. 3 Isoelectric point as a function of average number of acrylic acid units at $\mathrm{pH}=7$. Used co-oligomers: (square) $\mathrm{BA}_{4}-\mathrm{co}-\mathrm{AA}_{8}$, (triangle) $\mathrm{BA}_{16}-c o-\mathrm{AA}_{32}$, (diamond) $\mathrm{BA}_{5}-c o-\mathrm{AA}_{5}$, and (star) $\mathrm{BA}_{20}-c o-\mathrm{AA}_{20}$

at $70{ }^{\circ} \mathrm{C}$ for another two hours. During polymerization, samples were collected to analyse molar mass and particle size distributions.

\section{Results and discussion}

\section{Adsorption studies}

The vesicle templates were prepared using the surfactant DODAB and cryo-TEM was used to characterize the resulting morphology (Fig. 1).

As can be seen from Fig. 1, unilamellar vesicles were produced with a $Z$-average diameter $\left(D_{\mathrm{z}}\right)$ of $160 \mathrm{~nm}$ and a zeta potential of $+39 \mathrm{mV}$ at $\mathrm{pH} \approx 7$.

Anionic co-oligomers with different combinations of acrylic acid and butyl acrylate units were synthesized using ATRP and the resulting characteristics are summarized in Table 1.

In this work, we studied the adsorption of these cooligomers on the DODAB vesicles and the stoichiometric charge ratio parameter $(\xi)$ was used to express the final composition of the mixture (Eq. 1):

$$
\xi=\frac{N_{\text {acid }} \times[\text { co }- \text { oligomer }]}{[\mathrm{DODAB}]}
$$

Fig. 4 Cryo-TEM images of ATRP macroinitiator stabilized DODAB vesicles. Used cooligomers: (a) $\mathrm{BA}_{4}-\mathrm{co}-\mathrm{AA}_{8}$, (b) $\mathrm{BA}_{16}-\mathrm{co}-\mathrm{AA}_{32}$, (c) $\mathrm{BA}_{5}-\mathrm{Co}-\mathrm{AA}_{5}$, and (d) $\mathrm{BA}_{20}-\mathrm{CO}-\mathrm{AA}_{20}$
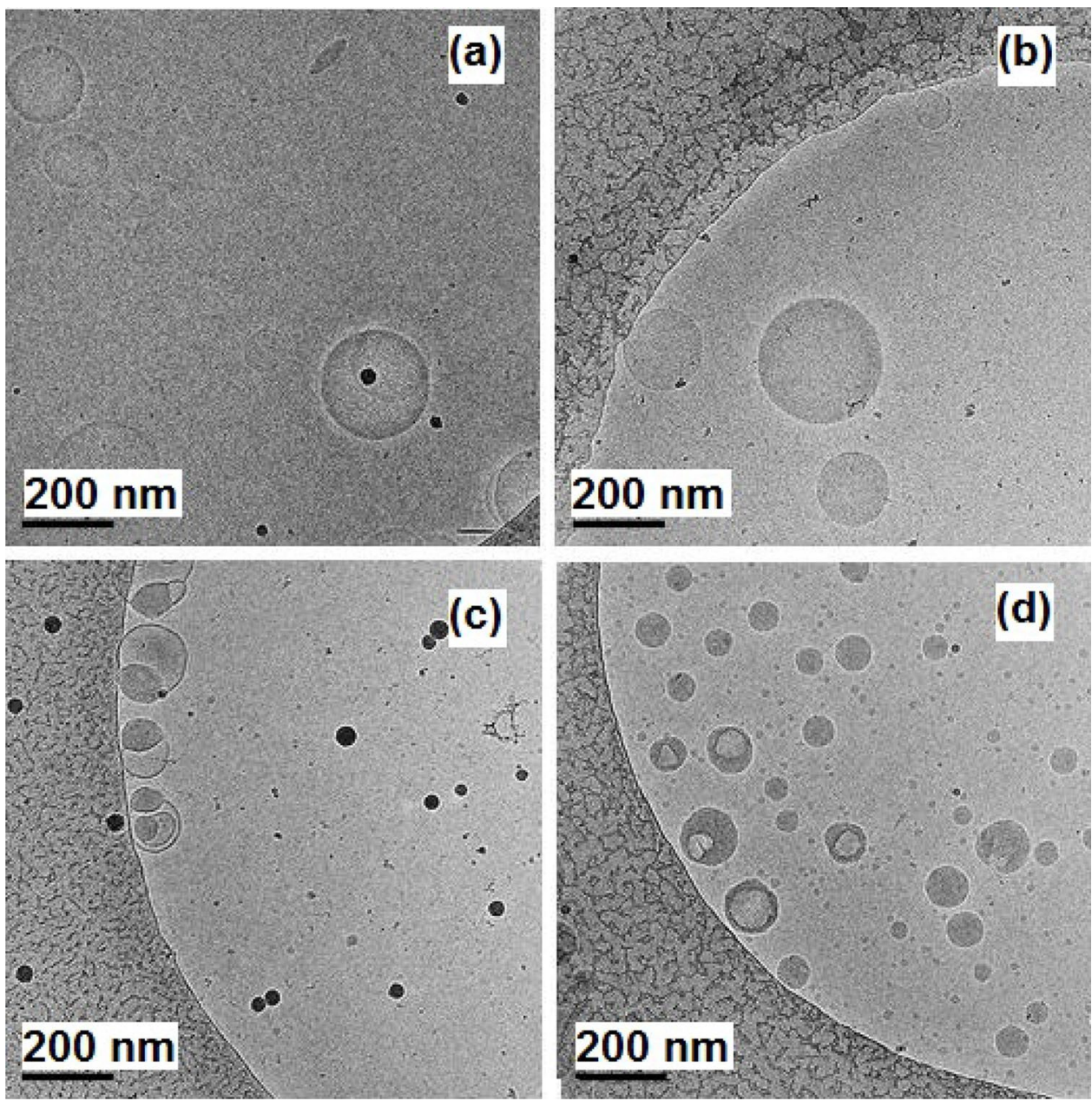
where $N_{\text {acid }}$ is the total number of acrylic acid units in ATRP macroinitiator and [co-oligomer] and [DODAB] are the molar concentrations of macroinitiator and vesicle forming molecules, respectively. It was previously stated that only DODAB molecules in the outer layer of the bilayer interacts with the ATRP macroinitiator [7]. This interaction was analyzed by the measurement of the zeta potential and the $D_{\mathrm{z}}$ (Fig. 2).

Similar to what was reported before [15], the addition of positively charged DODAB vesicles to negatively charged co-oligomer results in a charge inversion effect. As seen from Fig. 2a with an increasing charge ratio, the $D_{z}$ of the vesicles increases until aggregation occurs at the isoelectric point (iep), where the positive charge of the vesicles is totally neutralized by adsorbed ATRP macroinitiator. A further increase in the charge ratio parameter leads to a decrease in particle diameter and a plateau is reached at $\xi>1$ for the short cooligomers and at $\xi>2-3$ for the long co-oligomers.

As before, the zeta potential decreases with increasing charge ratio and becomes negative. The isoelectric points of the different co-oligomers were determined from these zeta-potential measurements (Table 1). When comparing the obtained values for different co-oligomers, it was noticed that charge ratio at the isoelectric point increased with increasing average number of acrylic acid units (Fig. 3).

The observed results can be explained by the affinity of longer hydrophilic chains to form more loop and tail structures on the surface of vesicles [23, 24]. As a consequence, a higher concentration of long chain co-oligomers is required to reach the iep.

Returning to the results on Fig. 2a, we noticed that a plateau was not reached in the cases of co-oligomers with equal BA/AA ratio. Since DODAB vesicles are soft templates, adsorption of ATRP macroinitiator may also provoke changes in the morphology of the initial vesicles. We therefore analyzed the effect of co-oligomer composition on morphology using cryo-TEM (Fig. 4).

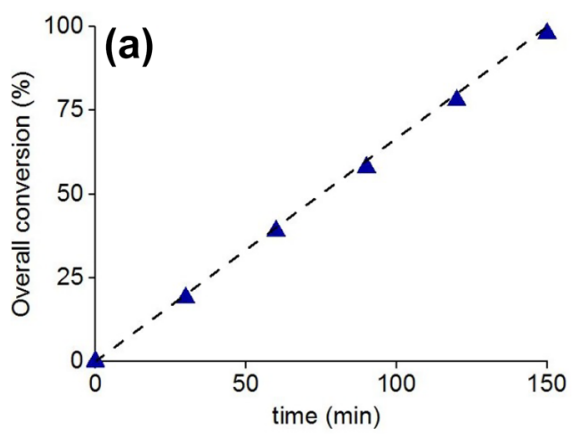

Fig. 6 a Overall conversion vs. time and $\mathbf{b}$ evolution of (triangle) Dz and (square) $\zeta$. Dashed line is monomer feeding profile. Optimized polymerization conditions: $\mathrm{V}=22.2 \mathrm{~mL}, \mathrm{~T}=70{ }^{\circ} \mathrm{C}, \xi=2,\left[\mathrm{Cu}^{2+}\right]$

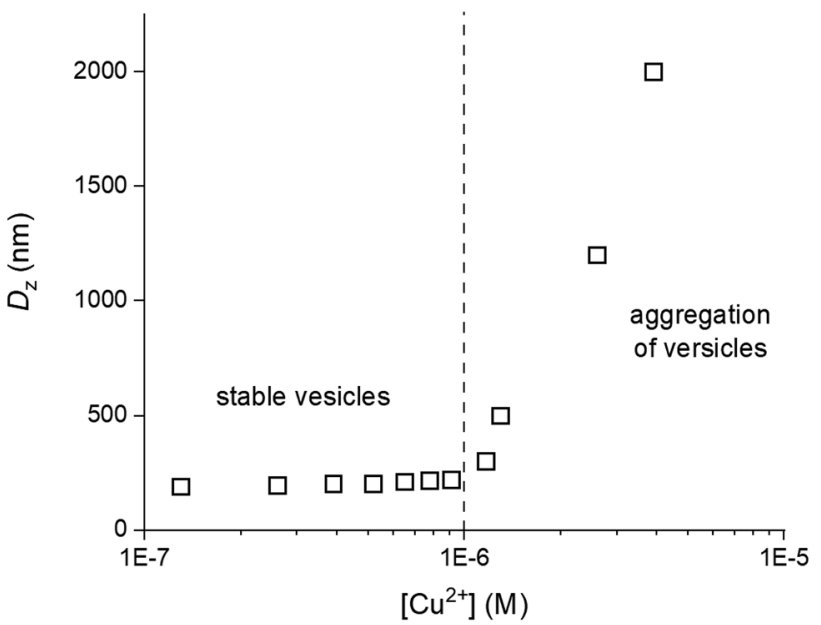

Fig. 5 Effect of $\left[\mathrm{Cu}^{2+}\right]$ on Dz of an ATRP macroinitiator-stabilized vesicles dispersion at $\xi=2$. Used co-oligomer: $\mathrm{BA}_{4}-\mathrm{Co}-\mathrm{AA}_{8}$

As can be seen from Fig. 4a to b, when co-oligomers with higher numbers of acrylic acid units were used, the initial morphology of the vesicles was not affected. However, in the situations with equal ratio of BA/AA (Fig. 4c-d), different morphologies were observed. The reason for difference in the morphology is due to the hydrophilic-hydrophobic balance of the ATRP copolymer and the fluidity of the DODAB bilayer. The segregation observed in the Fig. 4c and d is likely caused by an inadequate number of charged units to provide adhesion of the ATRP copolymer chains to the vesicle surface. As a result, dynamic distortion in the bilayer structure occurred.

From these results, it seems that in order to maintain the initial vesicle morphology, an excess of acrylic acid units is required. Interestingly, when using co-oligomers with higher AA units, no effect of the co-oligomer chain length was found. For further experiments, $\mathrm{BA}_{4}-\mathrm{co}-\mathrm{AA}_{8}$ was chosen and the polymerization was conducted at $\xi=2$. 
Fig. 7 Cryo-TEM images of final polymer-vesicle latex particles. Conditions as in Figure 6
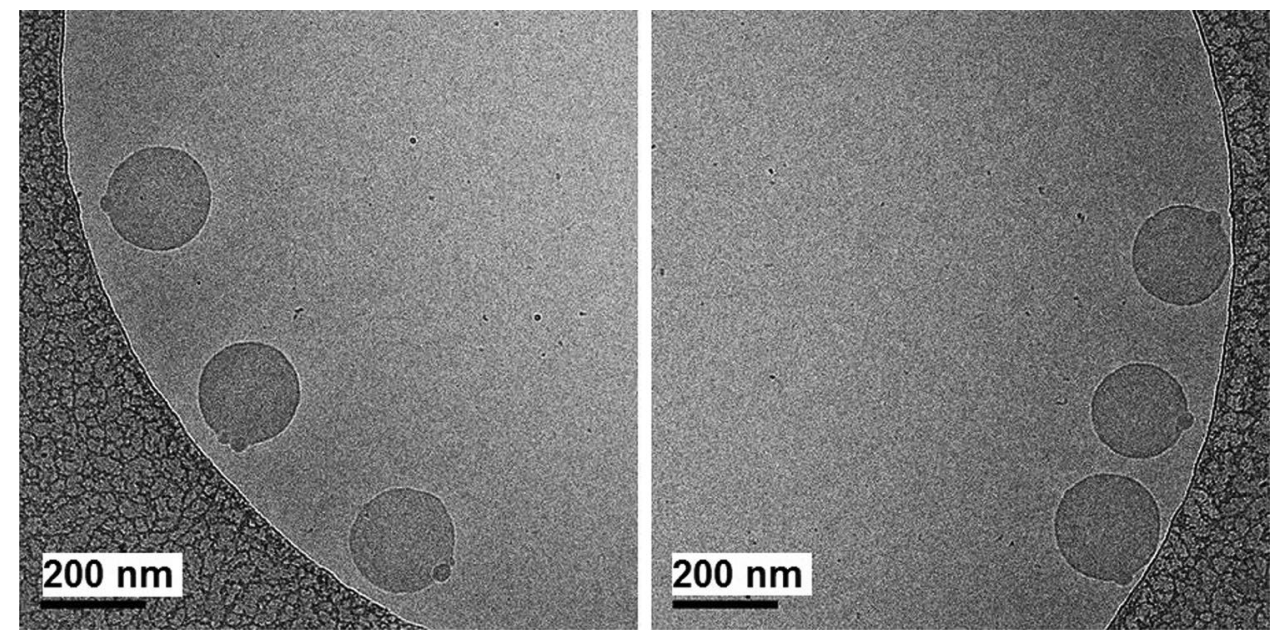

\section{Preparation and characterization of polymer-vesicle nanocomposites}

In our previous studies on the encapsulation of Gibbsite encapsulation using ATRP, it was found that the $\left[\mathrm{Cu}^{2+}\right]$ could have a profound effect on the colloidal stability [21]. Therefore, the effect of the $\left[\mathrm{Cu}^{2+}\right]$ on colloidal stability of the vesicles was investigated and the results are shown in Fig. 5.

As expected, the results from Fig. 5 clearly demonstrate the effect of $\left[\mathrm{Cu}^{2+}\right]$ on colloidal stability. Aggregation of vesicles occurs at $\left[\mathrm{Cu}^{2+}\right]>1.2 \times 10^{-6} \mathrm{M}$. We therefore conducted further experiments below this concentration.

Encapsulation experiments were carried out by using starved-feed conditions. Figure 6 shows the conversion and particle size evolution as a function of the added monomer amount during the polymerization.

Figure 6a clearly shows that the overall conversion follows the monomer feeding profile, indicating truly starvedfeed conditions. From the size measurements, increase on around $20 \mathrm{~nm}$ was observed over the polymerization time (Fig. 6b). The observed decrease in the $\zeta$-potential can be attributed to the chain extension from the macroinitiator and an increase in surface area to be stabilized.

We characterized the morphology of the obtained polymervesicle latex particles using cryo-TEM (Fig. 7). It should be noted that for the vesicle-templated ATRP-mediated polymerization, a relatively low monomer concentration was used as compared to previous work $[7,8,20]$. The reason was to avoid the formation of solid particles as seen in previous studies by Ali et al. [7, 8, 20].

These solid particles are likely to be separate latex particles formed by homogenous nucleation.

Cryo-TEM characterization of the obtained composite latex particles showed growth of polymer from one side of the vesicle, a so-called "parachute" structure. In the majority of particles (92\%), one small polymer bead was confined to the vesicle. Only a small fraction of the obtained composite particles consisted of 2-4 beads per vesicle.

\section{Effect of monomer composition}

Previously, the effect of monomer composition (100\% MMA and MMA:BA $=7: 3 \mathrm{w} / \mathrm{w}$ ) on polymer-vesicle particle
Fig. 8 Cryo-TEM image of final polymer-vesicle latex particles obtained using a monomer feed of a $100 \%$ MMA and $\mathbf{b}$ MMA:BA:EGDMA = 10:1:1 w/w. Other conditions as in Fig. 6. Secondary particles observed on Fig. 8b are ice artifacts from cryo-TEM imaging
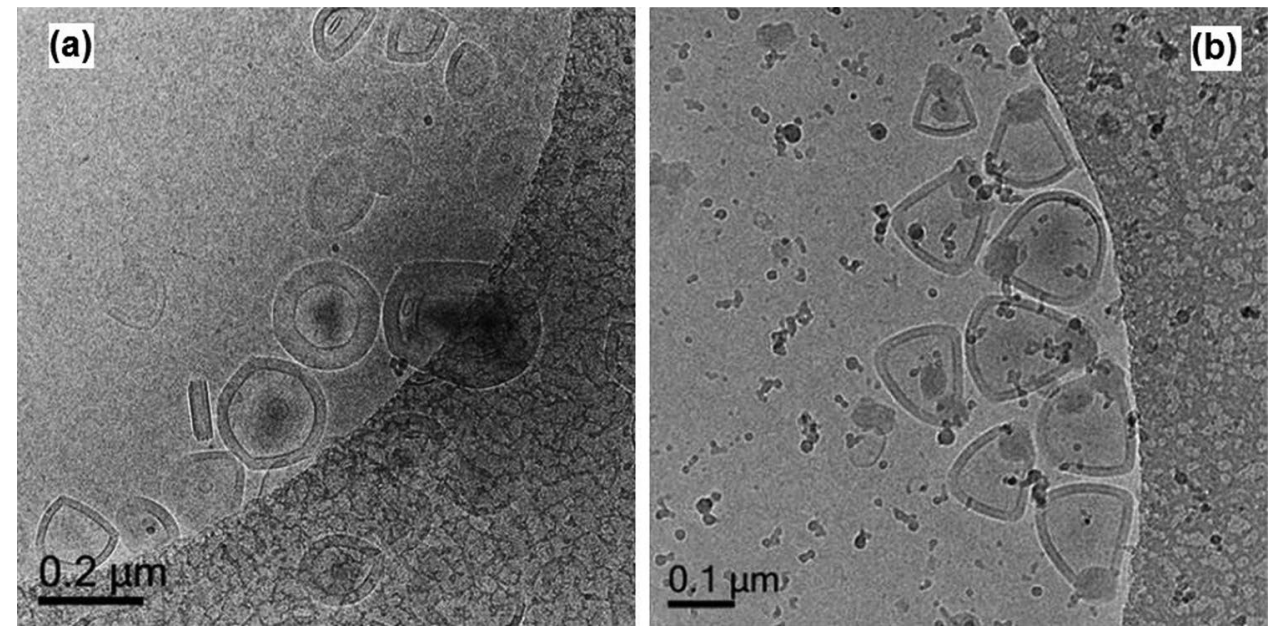
Table 2 Characterization of the composite latexes $^{a}$

\begin{tabular}{|c|c|c|c|c|c|c|}
\hline & \multicolumn{3}{|c|}{ After adsorption } & \multicolumn{3}{|c|}{ After polymerization } \\
\hline & $D_{\mathrm{z}}(\mathrm{nm})^{\mathrm{b}}$ & PDI & $\zeta(\mathbf{m V})$ & $D_{\mathrm{z}}(\mathrm{nm})$ & PDI & $\zeta(\mathbf{m V})$ \\
\hline $\mathrm{BA}_{4}-c o-\mathrm{AA}_{8}$ & 167 & 0.12 & -57 & 180 & 0.07 & -42 \\
\hline $\mathrm{BA}_{16}-\mathrm{co}-\mathrm{AA}_{32}$ & 184 & 0.08 & -63 & 207 & 0.07 & -46 \\
\hline $\mathrm{BA}_{5}-\mathrm{co}-\mathrm{AA}_{5}$ & 177 & 0.20 & -59 & 208 & 0.26 & -43 \\
\hline $\mathrm{BA}_{20}-c o-\mathrm{AA}_{20}$ & 140 & 0.21 & -60 & 153 & 0.11 & -47 \\
\hline
\end{tabular}

${ }^{a}$ Used monomer feed composition: $100 \%$ MMA. Initial DODAB vesicles dispersion: $D_{\mathrm{z}}=150 \mathrm{~nm}$, PDI value $=0.18$, and $\zeta=+39 \mathrm{mV}$. Other conditions as in Fig. 6

${ }^{\mathrm{b}}$ Estimated error in $D_{z}$ around $\pm 5 \mathrm{~nm}$ morphology was reported [7]. This was attributed to the glass transition temperature of the monomer composition. We therefore also studied the effect of monomer feed composition (100\% MMA and MMA:BA:EGDMA $=10: 1: 1$ $\mathrm{w} / \mathrm{w}$ ) on the polymer-vesicle hybrid particles morphology using cryo-TEM (Fig. 8).

As can be seen from Fig. 8a, a thick polymer layer was formed over the vesicle surface, resulting in the formation of a spherical hollow capsule. Meanwhile, feeding the second monomer composition (Fig. 8b) resulted in the accumulation of polymer in two locations of the vesicle: as a polymer particle on one side of the vesicle and a polymer layer over the whole vesicle surface. Observed capsule and protrusion structures clearly demonstrate the effect of monomer feed composition on the vesicle morphology.

\section{Effect of co-oligomer composition}

As shown above, the co-oligomer composition and chain length affect the adsorption and the resulting morphology of ATRP macroinitiator stabilized vesicle dispersion. This
Fig. 9 Cryo-TEM images of final polymer-vesicle latex particles obtained using a monomer feed of $100 \%$ MMA and different ATRP macroinitiators. Used co-oligomers: a $\mathrm{BA}_{4}-\mathrm{Co}-\mathrm{AA}_{8}$, b $\mathrm{BA}_{16}-\mathrm{Co}-\mathrm{AA}_{32}, \mathbf{c}$ $\mathrm{BA}_{5}-\mathrm{Co}-\mathrm{AA}_{5}$, and $\mathbf{d}$ $\mathrm{BA}_{20}-\mathrm{CO}-\mathrm{AA}_{20}$. Other conditions as in Fig. 6
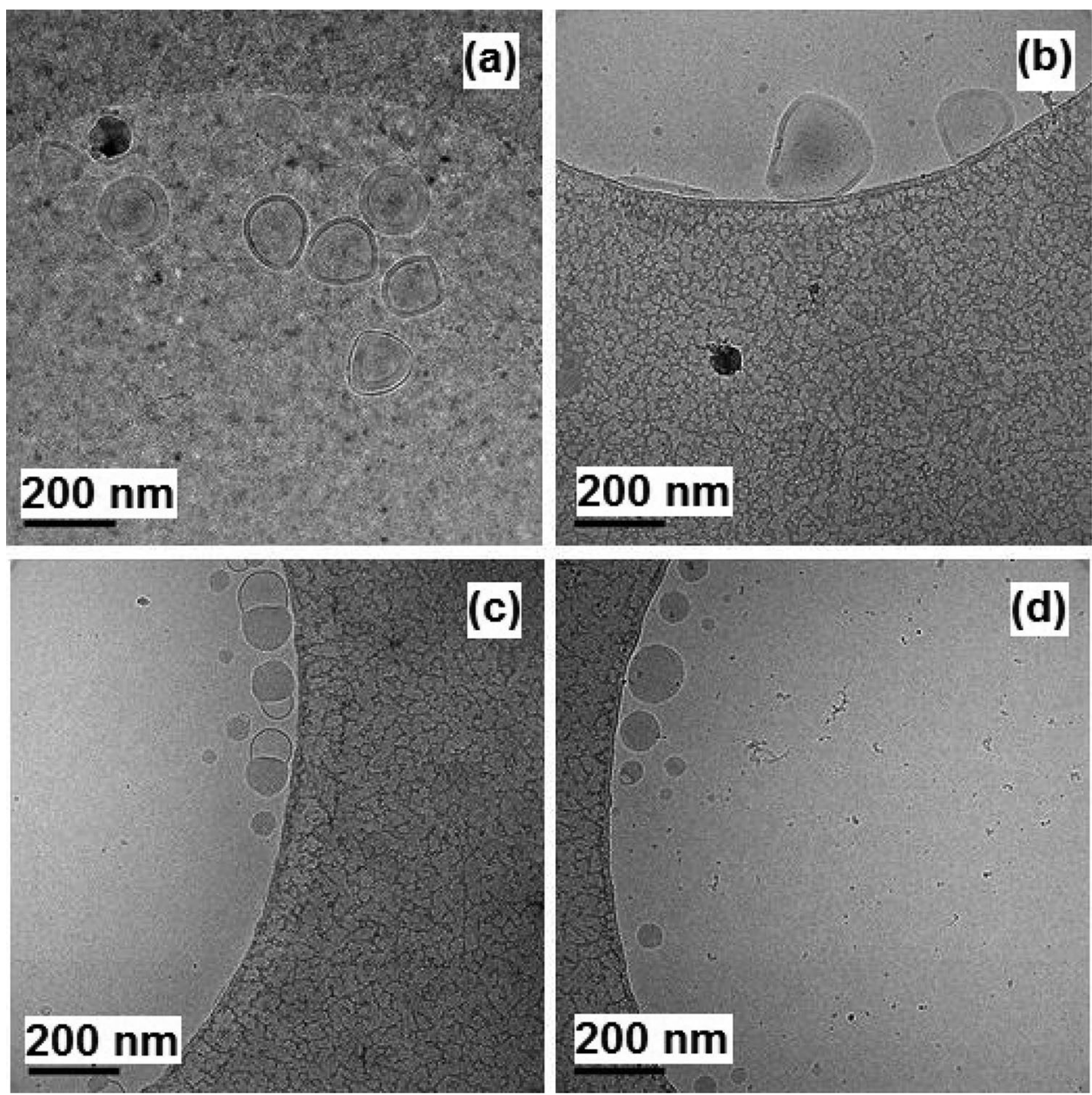
could potentially affect the polymerization process and the final morphology as was found in RAFT-based vesicle encapsulation studies [25, 26]. For that reason, we studied the effect of the co-oligomer composition and chain length on the morphology of the resulting composite latex particle. Characterization results of the latexes before and after polymerization are presented in Table 2 .

As can be seen from Table 2, the obtained polymervesicle latexes showed similar properties as those before (increase in $D_{\mathrm{z}}$ and decrease in $\zeta$-potential). The morphology of the obtained composite latex particles was analysed using cryo-TEM (Fig. 9).

As seen from Fig. 9a-b, polymeric nanocapsules were successfully obtained and the morphology did not depend on the chain length of the used co-oligomer. When co-oligomers with different lengths but the same BA/AA ratios were utilized (Fig. 9c-d), the same morphology, as after adsorption, was observed in the final latex particles. These results suggest that the morphology after polymerization is directly influenced by the morphology after adsorption as was previously also reported for RAFT polymerization [26].

The ATRP approach for vesicle polymerization fits in the overall pattern that has been seen for the RAFT approach, and the latter has been described in a recent review [27].

\section{Conclusions}

In this work, we have studied the synthesis of polymer vesicle hybrid particles using an ATRP-based method. The ATRP method introduces colloidal instability at too high copper concentrations but otherwise operates very similar to the previously reported RAFT-based approach. The morphologies of the obtained composite particles were similar to those previously obtained by the well-known RAFT-based approach. In both cases, cryo-TEM characterization revealed structures like nanocapsules and protrusions depending on the used monomer composition. Moreover, studies into cooligomer composition and chain length showed an effect on the initial vesicle structures during the adsorption and the resulting composite latex particles.

Funding The authors received financial support from the Euro-Asian Cooperation for Excellence and Advancement, Stichting Emulsion Polymerization and A*STAR Research Attachment Programme.

\section{Declarations}

Conflict of interest The authors declare no competing interests.

Open Access This article is licensed under a Creative Commons Attribution 4.0 International License, which permits use, sharing, adaptation, distribution and reproduction in any medium or format, as long as you give appropriate credit to the original author(s) and the source, provide a link to the Creative Commons licence, and indicate if changes were made. The images or other third party material in this article are included in the article's Creative Commons licence, unless indicated otherwise in a credit line to the material. If material is not included in the article's Creative Commons licence and your intended use is not permitted by statutory regulation or exceeds the permitted use, you will need to obtain permission directly from the copyright holder. To view a copy of this licence, visit http://creativecommons.org/licenses/by/4.0/.

\section{References}

1. Roling O, Wendeln C, Kauscher U, Seelheim P, Galla H-J, Ravoo BJ (2013) Layer-by-layer deposition of vesicles mediated by supramolecular interactions. Langmuir 29:10174-10182. https:// doi.org/10.1021/la4011218

2. van Herk AM, Forcada J, Pastorin G (2016) Controlled release systems: advances in nanobottles and active nanoparticles. Pan Stanford Publishing, Singapore

3. Pucci C, Salvia A, Ortore MG, La Mesa C (2013) The DODABAOT-water system: vesicle formation and interactions with salts or synthetic polyelectrolytes Soft Matter 9:9000-9007. https://doi. org/10.1039/C3SM51624E

4. Tang Z, Wang Y, Podsiadlo P, Kotov NA (2006) Biomedical applications of layer-by-layer assembly: from biomimetics to tissue engineering. Adv Mater 18:3203-3224. https://doi.org/10.1002/ adma.200600113

5. Zhu Y, Yang B, Chena S, Du J (2017) Polymer vesicles: mechanism, preparation, application, and responsive behavior Prog Polym Sci 64:1-22. https://doi.org/10.1016/j.progpolymsci.2015. 05.001

6. Meier W (1999) Nanostructure synthesis using surfactants and copolymers Curr Opin Colloid. Interface Sci 4:6-14. https://doi. org/10.1016/S1359-0294(99)00006-0

7. Ali SI, Heuts JP, van Herk AM (2010) Controlled synthesis of polymeric nanocapsules by RAFT-based vesicle templating. Langmuir 26:7848-7858. https://doi.org/10.1021/la904709c

8. Ali SI, Heuts JP, van Herk AM (2011) Vesicle-templated pHresponsive polymeric nanocapsules. Soft Matter 7:5382-5390. https://doi.org/10.1039/C1SM05266G

9. Jung M, Hubert DHW, van Veldhoven E, Frederik P, van Herk AM, German AL (2000) Vesicle-polymer hybrid architectures 1: a full account of the parachute architecture Langmuir 16:31653174. https://doi.org/10.1021/la991233a

10. Kunitake T, Okahata Y (1977) A totally synthetic bilayer membrane. J Am Chem Soc 99:3860-3861. https://doi.org/10.1021/ ja00453a066

11. Cuomo F, Lopez F, Miguel MG, Lindman B (2010) Langmuir 26:10555-10560. https://doi.org/10.1021/la100584b

12. Kaminski GAT, Sierakowski MR, Pontarolo R, dos Santos LA, de Freitas RA (2016) Carbohydr Polym 140:129-135. https://doi. org/10.1016/j.carbpol.2015.12.014

13. Guzmán E, Mateos-Maroto A, Ruano M, Ortega F, Rubio RG (2017) Layer-by-Layer polyelectrolyte assemblies for encapsulation and release of active compounds. Adv Colloid Interface Sci 249:290-307. https://doi.org/10.1016/j.cis.2017.04.009

14. Pastoriza-Santos I, Schöler B, Caruso F (2001) Core-shell colloids and hollow polyelectrolyte capsules based on diazoresins Adv Funct Mater 11:122-128.

15. Ali SI, Heuts JPA, Hawkett BS, van Herk AM (2009) Polymer encapsulated gibbsite nanoparticles: efficient preparation of anisotropic composite latex particles by RAFT-based starved feed emulsion polymerization. Langmuir 25:10523-10533. https://doi. org/10.1021/la9012697 
16. Nguyen D, Zondanos HS, Farrugia JM, Serelis AK, Such CH, Hawkett BS (2008) Pigment encapsulation by emulsion polymerization using macro-raft copolymers. Langmuir 24:2140-2150. https://doi.org/10.1021/la7027466

17. Nguyen D, Such C, Hawkett BS (2012) Polymer-TiO $\mathrm{T}_{2}$ composite nanorattles via RAFT-mediated emulsion polymerization J Polym Sci A Polym Chem 50:346-352. https://doi.org/10.1002/pola. 25038

18. Huynh VT, Nguyen D, Such CH, Hawkett BS (2015) Polymer coating of graphene oxide via reversible addition-fragmentation chain transfer mediated emulsion polymerization J Polym Sci A Polym Chem 53:1413-1421. https://doi.org/10.1002/pola.27596

19. Nguyen D, Such CH, Hawkett BS (2013) Polymer coating of carboxylic acid functionalized multiwalled carbon nanotubes via reversible addition-fragmentation chain transfer mediated emulsion polymerization. J Polym Sci A Polym Chem 51:250-257. https://doi.org/10.1002/pola.26389

20. Loiko OP, van Herk AM, Ali SI, Burkeev MZh, Tazhbayev YM, Zhaparova LZh (2013) Controlled release of Capreomycin sulfate from $\mathrm{pH}$-responsive nanocapsules e-Polymers 13:189-202. https://doi.org/10.1515/epoly-2013-0118

21. Loiko OP, Spoelstra AB, Van Herk AM, Meuldijk J, Heuts JPA (2016) An ATRP-based approach towards water-borne anisotropic polymer-Gibbsite nanocomposites, Polym Chem 7:3383-3391 https://doi.org/10.1039/C6PY00225K

22. Colombani O, Ruppel M, Schubert F, Zettl H, Pergushov DV, Müller AHE (2007) Synthesis of poly( $n$-butyl acrylate)-block-poly(acrylic acid) diblock copolymers by ATRP and their micellization in water macromolecules 40:4338-4350. https://doi.org/10.1021/ma0609578

23. Quemeneur F, Rinaudo M, Maret G, Pépin-Donat B (2010) Characterization of the adsorption of poly(acrylamide), poly(4methoxystyrene), and poly(acrylic acid) on aluminum oxide by inelastic electron tunneling spectroscopy Soft Matter 6:44714481. DOI. https://doi.org/10.1039/C0SM00154F

24. Colletti RF, Gold HS, Dybowski C (1987) Characterization of the adsorption of poly(acrylamide), poly(4-methoxystyrene), and poly(acrylic acid) on aluminum oxide by inelastic electron tunneling spectroscopy Appl Spectrosc 41:1185-1189.

25. Moradi M-A, Bomans PHH, Jackson AW, Van Herk AM, Heuts JPA (2018) A quantitative cryoTEM study on crosslinked nanocapsule morphology in RAFT-based vesivle polymerization. Eur Polym J 108:329-336. https://doi.org/10.1016/j.eurpolymj.2018. 09.008

26. Moradi M-A, Tempelaar S, Van Herk AM, Heuts JPA (2019) Morphology control of liposome-RAFT oligomer precursors to complex polymer nanostructures. Macromolecules 52:9476-9483. https://doi.org/10.1021/acs.macromol.9b02182

27. van Herk AM (2020) Vesicle-templated polymerization, a review. Biomacromol 21:4379-4387. https://doi.org/10.1021/acs.biomac. 0c00558

Publisher's Note Springer Nature remains neutral with regard to jurisdictional claims in published maps and institutional affiliations. 